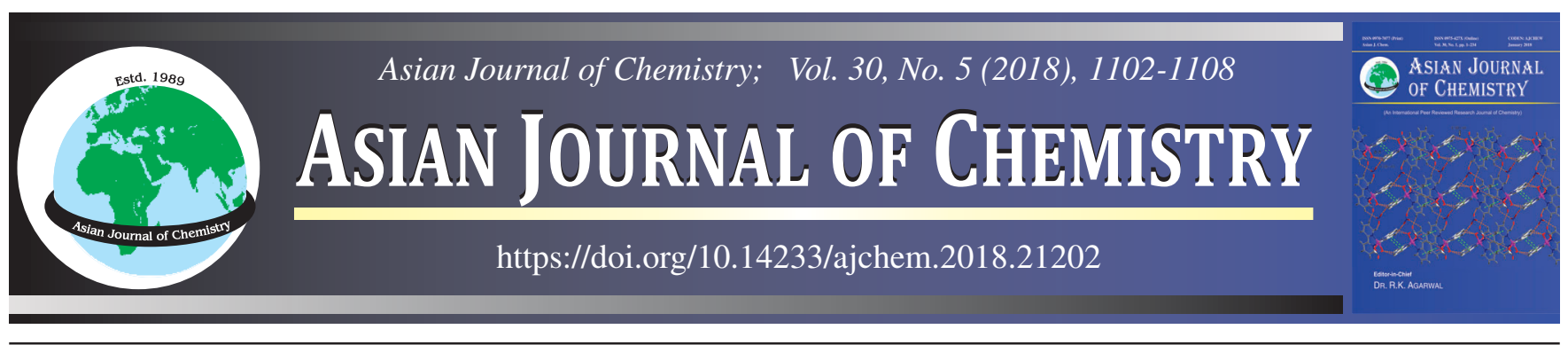

\title{
Synthesis, Characterization, Stereochemistry and Biological Evaluation of Novel Cyclohexanol Derivatives
}

Sayeed Mukhtar ${ }^{*}$, Meshari A. Alsharif, Mohammed I. Alahmdi and Humaira Parveen

Department of Chemistry, Faculty of Science, University of Tabuk, Tabuk-71491, Kingdom of Saudi Arabia

*Corresponding author: E-mail: sayeed_mukhtar@hotmail.com

Received: 26 December 2017;

Accepted: 18 February 2018;

Published online: 29 March 2018;

AJC-18849

This research includes the synthesis of 1,5-diketone from chalcone by Michael addition of $p$-chloroacetophenone in the presence of $\mathrm{NaOH}$ (molar ratio, 1:1:10), which led to the formation of a novel cyclohexanol derivative as a side product. The above reaction was repeated with different molar ratio of chalcone-acetophenone-sodium hydroxide to set the optimum condition for maximum yield of the novel cyclohexanol derivative. The dehydration of cyclohexanol using catalytic amount of $p$-TsOH produced quantitative yield of corresponding cyclohexene with $\beta, \gamma$-unsaturation instead of apparently more stable $\alpha, \beta$-unsaturation. The compounds were well characterized by spectroscopic techniques and elemental analysis. Their stereochemistry is discussed and newly synthesized compounds are screened for anticancer and antimicrobial activities.

Keywords: Chalcones, Cyclohexanol derivatives, Stereochemistry, in vitro Anticancer activity, in vitro Antimicrobial activity.

\section{INTRODUCTION}

Recently, a considerable amount of research on biologically active cyclohexanol derivatives has been reported [1-6] and has attracted growing interest of medicinal chemists. An elaborative literature survey on cyclohexanol derivatives has revealed that they exhibited diverse biological activities such as acetylcholine-storage-blocking activities [7], analgesic [8], antidepressant [9], cardio-inhibitory effects [10] and also have been reported as potent microbial agents [11].

In view of these observations and as part of our program in search of biologically active compounds with sulphur and nitrogen containing heterocycles [12-18], we report here the synthesis, characterization, stereochemistry, anticancer and antimicrobial studies of cyclohexanol derivatives.

\section{EXPERIMENTAL}

Reagents and solvents were of commercial grade and used without further purification. Melting points were determined on a Koffler hot-plate apparatus and are uncorrected. Elemental analysis $(\mathrm{C}, \mathrm{H}, \mathrm{N})$ were undertaken using an Elemental analyzer and within $\pm 0.4 \%$ of the calculated values. IR spectra were recorded on a Perkin-Elmer 621 spectrophotometer while ${ }^{1} \mathrm{H}$ and ${ }^{13} \mathrm{C}$ NMR spectra were recorded on a Varian Unity 400 spectrometer at $400 \mathrm{MHz}$ and $100 \mathrm{MHz}$, respectively in acetone- $d_{6}$ with TMS as the internal standard. DCI-mass spectra were recorded in a Ribermag R10-10B quadrupole mass spectrometer. Column chromatography was performed on silica gel (60-120 mesh).

4'-Chloro-4-methoxychalcone (1a) and 4,4'-dichlorochalcone (1b) were synthesized according to the published method [19].

General procedure for the synthesis of 1,5-diketones and cyclohexanol derivatives: A mixture of chalcone (1a) (500 mg, $1.83 \mathrm{mmol}$ ), $p$-chloroacetophenone $(0.24 \mathrm{~mL}, 283$ $\mathrm{mg}, 1.83 \mathrm{mmol}$ ) in an aqueous ethanolic solution of $\mathrm{NaOH}$ (7 mL, $\left.\mathrm{H}_{2} \mathrm{O}-\mathrm{EtOH}, 1: 1\right)(734 \mathrm{mg}, 18.3 \mathrm{mmol}$ ) was heated on a water bath for $5 \mathrm{~h}$ monitoring the progress of the reaction by TLC at every $15 \mathrm{~min}$. After completion, the reaction mixture was extracted with ethyl acetate, washed with water and the organic phase dried over $\mathrm{Na}_{2} \mathrm{SO}_{4}$. The solvent was distilled off under reduced pressure to a semi-solid residue which on column chromatography (silica gel, petroleum ether $\left(60-80^{\circ} \mathrm{C}\right)-$ diethyl ether, $6: 4 \mathrm{v} / \mathrm{v}$ ) followed by crystallization from $\mathrm{C}_{6} \mathrm{H}_{6}-\mathrm{EtOAc}$ yielded compounds $\mathbf{2 a}$ and $\mathbf{3} \mathbf{a}$ as white crystalline needles.

1,5-Bis(4-chlorophenyl)-3-(4-methoxyphenyl)pentan1,5-dione (2a): Yield: 68\%; m.p $80{ }^{\circ} \mathrm{C}, \mathrm{R}_{\mathrm{f}} 0.67$ (benzenediethyl ether, 9.5:0.5 v/v). IR $\left(\mathrm{KBr}, \mathrm{cm}^{-1}\right): 1675 \mathrm{v}(\mathrm{C}=\mathrm{O}), 1610$, $1580 v$ (phenyl), 1510, 1400, 1350, 1250, 1170, 1075, 980, 810; ${ }^{1} \mathrm{H}$ NMR (400 MHz, acetone- $d_{6}$ ): $\delta 3.41$ (dd, 2H, H-2up/ 4up, $J=16.94 \mathrm{~Hz}, J=7.48 \mathrm{~Hz}) ; 3.51(\mathrm{dd}, 2 \mathrm{H}, \mathrm{H}-2 \mathrm{dn} / 4 \mathrm{dn}, J=$ $16.94 \mathrm{~Hz}, J=6.56 \mathrm{~Hz}$ ); 3.98 (br p, $1 \mathrm{H}, \mathrm{H}-3, J \approx 7 \mathrm{~Hz}$ ); 3.70 (s, $\left.3 \mathrm{H}, \mathrm{H}-\mathrm{OCH}_{3}\right) ; 7.25$ (d, 2H, H-Ar'-2,6, J = 8.85 Hz); $6.78(\mathrm{~d}, 2 \mathrm{H}$, 
H-Ar'-3,5, J = 8.85 Hz); 7.98 (d, 4H, H-Ar-2, 6, J = 8.85 Hz); 7.49 (d, $4 \mathrm{H}, \mathrm{H}-\mathrm{Ar}-3,5, J=8.85 \mathrm{~Hz}) ;{ }^{13} \mathrm{CNMR}(100 \mathrm{MHz}$, acetone$\left.d_{6}\right): \delta 45.70(\mathrm{C}-2 / 4), 37.25(\mathrm{C}-3), 55.42\left(\mathrm{C}-\mathrm{OCH}_{3}\right) 129.51(\mathrm{C}-$ $\left.\mathrm{Ar}^{\prime}-2,6\right), 114.57$ (C-Ar'-3,5), 130.62 (C-Ar-2,6), 129.57 (C-Ar3,5), 136.96 (C-Ar'-1), 159.27 (C-Ar'-4), 136.86 (C-Ar-1), 139.45 (C-Ar-4), 198.06(C-CO), MS (DCI): 431/433/435 (M+ + 1); Anal. Calcd for $\mathrm{C}_{24} \mathrm{H}_{20} \mathrm{O}_{3} \mathrm{Cl}_{2}$ : C, 67.46; H, $4.72 \%$; Found: $\mathrm{C}$, $67.39 ; \mathrm{H}, 4.70 \%$.

2,4-Bis(4-chlorobenzoyl)-1-(4-chlorophenyl)-3,5-bis(4methoxyphenyl)cyclohexan-1-ol (3a): Yield: 6.6\%, m.p. 232 ${ }^{\circ} \mathrm{C}, \mathrm{R}_{\mathrm{f}} 0.42$ (benzene-diethyl ether, 9.5:0.5 v/v). IR $\left(\mathrm{KBr}, \mathrm{cm}^{-1}\right)$ : $3480 v(\mathrm{OH}) ; 1665,1640 v(\mathrm{C}=\mathrm{O}) ; 1610,1585 v$ (phenyl), 1510, $1400,1250,1175,1085,1025,1005,820$. The $\delta_{\mathrm{H}}$ and $\delta_{\mathrm{C}}$ values are given in Tables 1 and 2. MS (ApcI): $m / z, 681 / 683 / 685 / 687$ $\left[\mathrm{M}^{+}+1-\mathrm{H}_{2} \mathrm{O}\right]^{+}$.

The above reaction was also carried out with different molar ratios of chalcone 1a and acetophenone in the presence of 10 equiv. $\mathrm{NaOH}(10 \%)$ as described above which furnished compounds $\mathbf{2 a}$ and $\mathbf{3 a}$ in varying yields.

Further, Michael reaction of chalcone 1a with the isolated 1,5-diketone 2a was also conducted as follows: A mixture of chalcone 1a (400 mg, $1.46 \mathrm{mmol})$ and 1,5-diketone 2a (500 $\mathrm{mg}, 1.17 \mathrm{mmol}$ ) in $\mathrm{NaOH}$ solution $\left(5.0 \mathrm{~mL}, \mathrm{H}_{2} \mathrm{O}-\mathrm{EtOH}, 1: 1\right)$ $(470 \mathrm{mg}, 11.7 \mathrm{mmol})$ was heated on a water bath for $5 \mathrm{~h}$. The products on usual workup and crystallization afforded compound 3a as white crystalline globules, $570 \mathrm{mg}$ (69.6\%), m.p. $232{ }^{\circ} \mathrm{C}, \mathrm{R}_{\mathrm{f}} 0.42$ (benzene-diethyl ether, 9.5:0.5 v/v).

1,3,5-Tris(4-chlorophenyl)pentan-1,5-dione (2b): Yield: $72 \%$; m.p $110^{\circ} \mathrm{C}, \mathrm{R}_{\mathrm{f}} 0.85$ (benzene-diethyl ether 9.5:0.5 v/v); IR $\left(\mathrm{KBr}, \mathrm{cm}^{-1}\right): 1680 v(\mathrm{C}=\mathrm{O}), 1610,1585 v$ (phenyl), 1520, 1400, $1350,1260,1165,1060,980,810 .{ }^{1} \mathrm{H}$ NMR $(400 \mathrm{MHz}$, acetone$\left.d_{6}\right): 3.49(\mathrm{dd}, 2 \mathrm{H}, \mathrm{H}-2 \mathrm{up} / 4 \mathrm{up}, J=17.24 \mathrm{~Hz}, J=7.63 \mathrm{~Hz}) ; 3.59$ (dd, $2 \mathrm{H}, \mathrm{H}-2 \mathrm{dn} / 4 \mathrm{dn}, J=17.24 \mathrm{~Hz}, J=6.41 \mathrm{~Hz}$ ); 4.05 (br p, $1 \mathrm{H}, \mathrm{H}-3, \mathrm{~J} \approx 7 \mathrm{~Hz}$ ); 7.26 (d, 2H, H-Ar'-2,6, J=8.54 Hz); 7.40 (d, 2H, H-Ar'-3,5, J = 8.54 Hz); 8.00 (d, 4H, H-Ar-2,6, $J=$ $8.70 \mathrm{~Hz}) ; 7.52(\mathrm{~d}, 4 \mathrm{H}, \mathrm{H}-\mathrm{Ar}-3,5, J=8.70 \mathrm{~Hz}) .{ }^{13} \mathrm{C}$ NMR $(100$ $\mathrm{MHz}$, acetone- $\left.d_{6}\right)$ : 45.29 (C-2/4), 37.23 (C-3), 130.48 (C-Ar'-
2,6) 129.11 (C-Ar'-3,5), 130.64 (C-Ar-2,6), 129.64 (C-Ar-3,5), 132.44 (C-Ar'-1), 144.20 (C-Ar'-4), 136.74 (C-Ar-1), 139.60 (C-Ar-4), 197.77 (C-CO); MS (DCI): m/z 427/429/431/433 $\left(\mathrm{M}^{+}+1\right)$; Anal. Calcd for $\mathrm{C}_{23} \mathrm{H}_{17} \mathrm{O}_{2} \mathrm{Cl}_{3}: \mathrm{C}, 63.98 ; \mathrm{H}, 3.97 \%$. Found: C, 63.89; H, $3.96 \%$.

2,4-Bis(4-chlorobenzoyl)-1,3,5-tris(4-chlorophenyl)cyclohexan-1-ol (3b): Yield: $7.3 \%$; m.p. $242{ }^{\circ} \mathrm{C}, \mathrm{R}_{\mathrm{f}} 0.67$ (benzene-diethyl ether 9.5:0.5 v/v); IR $\left(\mathrm{KBr}, \mathrm{cm}^{-1}\right): 3475 \mathrm{v}(\mathrm{OH})$, $1665,1645 v(\mathrm{C}=\mathrm{O}), 1610,1580 v$ (phenyl), 1510, 1395, 1260, $1175,1085,1015,820 \mathrm{~cm} .{ }^{1} \mathrm{H}$ NMR $\left(400 \mathrm{MHz}\right.$, acetone- $\left.d_{6}\right)$ : $2.00\left(\mathrm{dd}, 1 \mathrm{H}, \mathrm{H}-6_{\mathrm{eq}}, J=3.66 \mathrm{~Hz}, J=13.74 \mathrm{~Hz}\right), 2.86(\mathrm{br} \mathrm{dt}, 1 \mathrm{H}$, $\left.\mathrm{H}-6_{\mathrm{ax}}, J=2.29 \mathrm{~Hz}, J=11-12 \mathrm{~Hz}, J=13.74 \mathrm{~Hz}\right), 3.92\left(\mathrm{brdt}, 1 \mathrm{H}, \mathrm{H}-5_{\mathrm{ax}}\right.$, $J \approx 11.10 \mathrm{~Hz}, J=11-12 \mathrm{~Hz}, J=3.66 \mathrm{~Hz}), 4.12\left(\right.$ br t $, 1 \mathrm{H}, \mathrm{H}-3_{\mathrm{ax}}$, $J \approx 11.45$ ); 4.82 (br t, $\left.1 \mathrm{H}, \mathrm{H}-4_{\text {ax }}, J \approx 11.14\right), 5.16\left(\mathrm{~d}, 1 \mathrm{H}, \mathrm{H}-2_{\text {ax }}\right.$, $J=11.60 \mathrm{~Hz}) ; 5.12(\mathrm{~d}, \mathrm{H}, \mathrm{H}-\mathrm{OH}, J=2.29 \mathrm{~Hz}) ; 6.91-7.73(\mathrm{br} \mathrm{m}$, $\mathrm{H}-5 \times$ Ar-rings). ${ }^{13} \mathrm{C}$ NMR $\left(100 \mathrm{MHz}\right.$, acetone- $\left.d_{6}\right): 46.51$ (C-6), 44.22 (C-5), 48.47 (C-3), 55.50a (C-4), 56.08 (C-2), 76.11 (C-1), 202.34 (C-CO), 205.33 (C-CO), 127.84-146.28 (C-5 × Ar-rings). MS (ApcI): $m / z, 689 / 691 / 693 / 695\left[\mathrm{M}^{+}+1-\mathrm{H}_{2} \mathrm{O}\right]^{+}$.

General procedure for the synthesis of 4,6-bis(4-chlorobenzoyl)-1-(4-chlorophenyl)-3,5-bis(4-methoxyphenyl)cyclohexene (4a): To a solution of compound $\mathbf{3 a}(100 \mathrm{mg}, 0.142$ $\mathrm{mmol})$ in dry benzene $(5 \mathrm{~mL}$ ) was added $p$-toluene sulphonic acid $(5 \mathrm{mg})$ and refluxed with stirring over an oil bath at $80^{\circ} \mathrm{C}$ for $3 \mathrm{~h}$. The reaction mixture was cooled, washed with water and the organic phase dried over $\mathrm{Na}_{2} \mathrm{SO}_{4}$. The solvent was distilled off under reduced pressure and the residue left on usual workup and crystallization from $\mathrm{C}_{6} \mathrm{H}_{6}$-EtOAc afforded compound $\mathbf{4 a}$ as white crystalline globules, $91 \mathrm{mg}(93.4 \%)$, m. p $218^{\circ} \mathrm{C}, \mathrm{R}_{\mathrm{f}} 0.60$ (benzene:diethyl ether, 9.5:0.5 v/v). IR $\left(\mathrm{KBr}, \mathrm{cm}^{-1}\right)$ : $2835 \mathrm{v}(\mathrm{C}-\mathrm{H})$, $1668 v(\mathrm{C}=\mathrm{O}), 1613,1587,1569 \mathrm{v}$ (phenyl), 1512,1488, 1462, 1440, 1399, 1305, 1287, 1254 1214, 1179, 1092, 1031, 1011, 971, $862,830,751,729,697$. The $\delta_{\mathrm{H}}$ and $\delta_{\mathrm{C}}$ values of compound $4 \mathbf{a}$ are given in Table-3. MS (ApcI): $m / z$ 681/683/685/687 $\left(\mathrm{M}^{+}+1\right)$.

4-Bis(4-chlorobenzoyl)-1,3,5-tris(4-chlorophenyl)cyclohexene (4b): Yield: $94.6 \%$; m.p. $254^{\circ} \mathrm{C}, \mathrm{R}_{\mathrm{f}} 0.90$ (benzenediethyl ether 9.5:0.5 v/v); IR (KBr, $\left.\mathrm{cm}^{-1}\right): 2858 \mathrm{v}(\mathrm{C}-\mathrm{H}), 1668$

TABLE-1

${ }^{1} \mathrm{H}$ NMR DATA OF 3a IN ACETONE- $d_{6}$

\begin{tabular}{|c|c|c|c|c|}
\hline H-nr & $\delta(\mathrm{ppm})$ & Integration & Multiplicity & $J(\mathrm{~Hz})+\mathrm{COSY}$ \\
\hline $66_{\text {eq }}$ & 1.97 & $1 \mathrm{H}$ & $\mathrm{Dd}$ & $J_{5 \text { ax }, 6 \mathrm{eq}} 3.66 ; J_{6 \mathrm{ax}, 6 \mathrm{eq} .} 13.73$ \\
\hline $6_{\mathrm{ax}}$ & 2.78 & $1 \mathrm{H}$ & br dt & $J_{6 \mathrm{ax}, \mathrm{OH}} 2.29 ; J_{5 \mathrm{ax}, 6 \mathrm{ax}} 11-12 ; J_{6 \mathrm{ax}, 6 \mathrm{eq}} 13.73$ \\
\hline $5_{\mathrm{ax}}$ & 3.86 & $1 \mathrm{H}$ & br dt & $\begin{array}{l}J_{4 \mathrm{ax}, 5 \mathrm{ax}} \approx 11.14 ; J_{5 \mathrm{ax}}, 6 \mathrm{ax} \\
11-12 ; J_{5 \mathrm{ax}, 6 \mathrm{eq}} 3.66\end{array}$ \\
\hline $3_{\mathrm{ax}}$ & 4.02 & $1 \mathrm{H}$ & br t & $J_{2 \mathrm{ax}, 3 \mathrm{ax}} \approx J_{3 \mathrm{ax}, 4 \mathrm{ax}} \approx 11.45$ \\
\hline $4_{\mathrm{ax}}$ & 4.71 & $1 \mathrm{H}$ & br t & $J_{3 \mathrm{ax}, 4 \mathrm{ax}} \approx J_{4 \mathrm{ax}}, 5 \mathrm{ax} \approx 11.14$ \\
\hline $2_{\text {ax }}$ & 5.08 & $1 \mathrm{H}$ & $\mathrm{D}$ & $J_{2 \mathrm{ax}, 3 \mathrm{ax}} 11.75$ \\
\hline $1-\mathrm{OH}$ & 5.14 & $1 \mathrm{H}$ & $\mathrm{D}$ & $J_{\mathrm{OH}, 6 \mathrm{ax}} 2.29$ \\
\hline $3-\left[\mathrm{Ar}^{\prime}-2,6\right]$ & 7.19 & $2 \mathrm{H}$ & d & $J_{\mathrm{Ar}^{\prime}-2,6, \mathrm{Ar}^{\prime}-3,5} 8.85$ \\
\hline $3-\left[\mathrm{Ar}^{\prime}-3,5\right]$ & 6.42 & $2 \mathrm{H}$ & d & 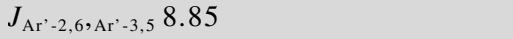 \\
\hline $3-\left[\mathrm{Ar}^{\prime}-\mathrm{OCH}_{3}\right]$ & 3.60 & $3 \mathrm{H}$ & $\mathrm{s}$ & \\
\hline $5-[$ Ar' $-2,6]$ & 7.26 & $2 \mathrm{H}$ & d & $J_{\mathrm{Ar}^{\prime}-2,6, \mathrm{Ar}^{\prime}-3,5} 8.70$ \\
\hline $5-\left[\mathrm{Ar}^{\prime}-3,5\right]$ & 6.66 & $2 \mathrm{H}$ & d & $J_{\mathrm{Ar}^{\prime}-2,6}, \mathrm{Ar}^{\prime}-3,58.70$ \\
\hline $5-\left[\mathrm{Ar}^{\prime}-\mathrm{OCH}_{3}\right]$ & 3.43 & $3 \mathrm{H}$ & $\mathrm{s}$ & \\
\hline 4-Ar-2,6 & 7.42 & $2 \mathrm{H}$ & d & $J_{\mathrm{Ar}-2,6, \mathrm{Ar}-3,5} 8.69$ \\
\hline 4-Ar-3,5 & 7.15 & $2 \mathrm{H}$ & d & $J_{\mathrm{Ar}-2,6, \mathrm{Ar}-3,5} 8.69$ \\
\hline $2-A r-2,6$ & 7.52 & $2 \mathrm{H}$ & d & $J_{\mathrm{Ar}-2,6, \mathrm{Ar}-3,5} 8.85$ \\
\hline $2-\mathrm{Ar}-3,5$ & 7.19 & $2 \mathrm{H}$ & d & $J_{\mathrm{Ar}-2,6, \mathrm{Ar}-3,5} 8.85$ \\
\hline $1-\mathrm{Ar}-2,6$ & 7.71 & $2 \mathrm{H}$ & d & $J_{\mathrm{Ar}-2,6, \mathrm{Ar}-3,5} 8.69$ \\
\hline $1-\mathrm{Ar}-3,5$ & 7.19 & $2 \mathrm{H}$ & d & $J_{\mathrm{Ar}-2,6, \mathrm{Ar}-3,5} 8.69$ \\
\hline
\end{tabular}




\begin{tabular}{|c|c|c|c|}
\hline \multicolumn{4}{|c|}{$\begin{array}{c}\text { TABLE-2 } \\
{ }^{13} \mathrm{C} \text { NMR DATA OF COMPOUND 3a IN ACETONE- } d_{6}\end{array}$} \\
\hline C-no. & $\delta(\mathrm{ppm})$ & HETCOR correlation with & Long-range HETCOR correlation with \\
\hline 6 & 47.03 & $\mathrm{H}-6_{\mathrm{ax}} ; \mathrm{H}-6_{\mathrm{eq}}$ & $1-\mathrm{OH}$ \\
\hline 5 & 43.94 & $\mathrm{H}-5_{\mathrm{ax}}$ & \\
\hline 3 & 48.38 & $\mathrm{H}-3_{\mathrm{ax}}$ & $\mathrm{H}-2_{\mathrm{ax}}, \mathrm{H}-4_{\mathrm{ax}}$ \\
\hline 4 & $56.45^{\mathrm{a}}$ & $\mathrm{H}-4_{\mathrm{ax}}$ & \\
\hline 2 & $56.36^{\mathrm{a}}$ & $\mathrm{H}-2_{\mathrm{ax}}$ & \\
\hline 1 & 76.25 & & \\
\hline $\mathrm{CO}$ & 203.03 & & \\
\hline $\mathrm{CO}$ & 205.95 & & \\
\hline $3-[$ Ar- 2,6$]$ & $128.84^{\mathrm{b}}$ & $\mathrm{H}-\{3-[\operatorname{Ar}-2,6]\}$ & \\
\hline $3-[$ Ar-3,5] & 114.28 & $\mathrm{H}-\{3-[\operatorname{Ar}-3,5]\}$ & \\
\hline $3-[\mathrm{Ar}-4]$ & 159.42 & & $\mathrm{H}-\left\{3-\left[\mathrm{Ar}^{\prime}-\mathrm{OCH}_{3}\right]\right\}$ \\
\hline $3-[\mathrm{Ar}-1]$ & 132.16 & & \\
\hline $3-\left[\mathrm{Ar}-\mathrm{OCH}_{3}\right]$ & $55.39^{c}$ & $\mathrm{H}-\left\{3-\left[\mathrm{Ar}-\mathrm{OCH}_{3}\right]\right\}$ & \\
\hline $5-[$ Ar- 2,6$]$ & 129.83 & $H-\{5-[A r-2,6]\}$ & $\mathrm{H}-5_{\mathrm{ax}}$ \\
\hline $5-[$ Ar- 3,5$]$ & 114.62 & $\mathrm{H}-\{5-[\mathrm{Ar}-3,5]\}$ & \\
\hline $5-[\mathrm{Ar}-4]$ & 159.42 & & $\mathrm{H}-\left\{5-\left[\mathrm{Ar}^{\prime}-\mathrm{OCH}_{3}\right]\right\}, \mathrm{H}-\left\{5-\left[\mathrm{Ar} r^{\prime}-2,6\right]\right\}$ \\
\hline $5-[\mathrm{Ar}-1]$ & 133.04 & & \\
\hline $5-\left[\mathrm{Ar}-\mathrm{OCH}_{3}\right]$ & $55.26^{\mathrm{c}}$ & $\mathrm{H}-\left\{5-\left[\mathrm{Ar}-\mathrm{OCH}_{3}\right]\right\}$ & \\
\hline $4-\operatorname{Ar}-2,6$ & 130.17 & $\mathrm{H}-\{4-[\mathrm{Ar}-2,6]\}]$ & \\
\hline $4-A r-3,5$ & 128.68 & $\mathrm{H}-\{4-[\mathrm{Ar}-3,5]\}$ & \\
\hline 4-Ar-1 & $135.53^{\mathrm{d}}$ & & \\
\hline $4-\mathrm{Ar}-4$ & $137.41^{\mathrm{d}}$ & & \\
\hline $2-A r-2,6$ & 130.64 & $\mathrm{H}-\{2-[\operatorname{Ar}-2,6]\}$ & \\
\hline $2-\mathrm{Ar}-3,5$ & $128.94^{\mathrm{b}}$ & $\mathrm{H}-\{2-[\mathrm{Ar}-3,5]\}$ & \\
\hline $2-\mathrm{Ar}-1$ & $138.34^{\mathrm{d}}$ & & \\
\hline $2-\mathrm{Ar}-4$ & $138.47^{\mathrm{d}}$ & & \\
\hline $1-\operatorname{Ar}-2,6$ & $128.84^{\mathrm{b}}$ & $\mathrm{H}-\{1-[\mathrm{Ar}-2,6]\}$ & \\
\hline $1-\mathrm{Ar}-3,5$ & $127.81^{\mathrm{b}}$ & $\mathrm{H}-\{1-[\mathrm{Ar}-3,5]\}$ & \\
\hline $1-\mathrm{Ar}-1$ & $139.69^{d}$ & & \\
\hline $1-\mathrm{Ar}-4$ & 146.73 & & \\
\hline
\end{tabular}

TABLE-3

${ }^{1} \mathrm{H}$ NMR SPECTRAL DATA OF COMPOUND 4a IN ACETONE- $d_{6}$

\begin{tabular}{|c|c|c|c|c|c|c|c|}
\hline H-no & $\delta(\mathrm{ppm})$ & Integration & Multiplicity & $J(\mathrm{~Hz})+\mathrm{COSY}$ & C-no & $\delta(\mathrm{ppm})$ & HETCOR \\
\hline 2 & 6.15 & $1 \mathrm{H}$ & $\mathrm{T}$ & $J_{6 \mathrm{ax}, 2,} J_{2,3 \mathrm{ax}} 2.14$ & 2 & 131.32 & $\mathrm{H}-2$ \\
\hline $3_{\mathrm{ax}}$ & 4.16 & $1 \mathrm{H}$ & br dq & $\begin{array}{l}J_{3 \mathrm{ax}, 4 \mathrm{ax}} \approx 11.10 ; J_{2,3 \mathrm{ax}} 2.14 ; J_{3 \mathrm{ax}}, \\
\text { 6pseud.ax} \approx 3.97\end{array}$ & 3 & 47.16 & $\mathrm{H}-3_{\text {pseud.ax }}$ \\
\hline $6_{\mathrm{ax}}$ & 5.49 & $1 \mathrm{H}$ & br dq & $\begin{array}{l}J_{5 \mathrm{ax}, 6 \mathrm{ax}} \approx 11.10 ; J_{3 \mathrm{ax}, 6 \mathrm{ax}} \approx 3.97 \\
J_{2,6} 2.14\end{array}$ & 6 & 55.15 & H- $6_{\text {pseud.ax }}$ \\
\hline $4_{\mathrm{ax}}$ & 4.57 & $1 \mathrm{H}$ & br t & $J_{5 \mathrm{ax}, 4 \mathrm{ax}} \approx J_{3 \mathrm{ax}, 4 \mathrm{ax}} \approx 11.10$ & 4 & 53.20 & $\mathrm{H}-4_{\mathrm{ax}}$ \\
\hline $5_{\mathrm{ax}}$ & 3.74 & $1 \mathrm{H}$ & br t & $J_{5 \mathrm{ax}, 6} \approx J_{4 \mathrm{ax}, 5 \mathrm{ax}} \approx 11.10$ & 5 & 50.42 & $\mathrm{H}-5_{\mathrm{ax}}$ \\
\hline $5 \times \mathrm{Ar}$ & $6.47-7.74$ & $20 \mathrm{H}$ & br m & - & $\mathrm{CO}$ & 204.10 & \\
\hline $\mathrm{H}-\mathrm{OCH}_{3}$ & 3.54 & $3 \mathrm{H}$ & s & - & $\begin{array}{l}\mathrm{CO} \\
5 \times \mathrm{Ar}\end{array}$ & $\begin{array}{l}206.83 \\
128.32-159.70\end{array}$ & \\
\hline
\end{tabular}

(C=O), 1589, 1569 v(phenyl), 1488, 1402, 1360, 1317, 1289, 1215, 1175, 1094, 1014, 981, 965, 910, 870, 842, 805, 761, 738, 720, 677. ${ }^{1} \mathrm{H}$ NMR (400 MHz, acetone- $\left.d_{6}\right): 6.17$ (t, $1 \mathrm{H}, \mathrm{H}-2_{\text {eq }}$, $J=2.14 \mathrm{~Hz}$ ); 4.23 (br dq, $1 \mathrm{H}, \mathrm{H}-3_{\mathrm{ax}}, J \approx 11.14 \mathrm{~Hz}, J \approx 2.14 \mathrm{~Hz}$, $J \approx 3.66 \mathrm{~Hz}$ ); 3.82 (br t, $1 \mathrm{H}, \mathrm{H}-5_{\mathrm{ax}}, J \approx 11.14 \mathrm{~Hz}$ ); 4.69 (br t, $1 \mathrm{H}$, $\mathrm{H}-4_{\mathrm{ax}}, J \approx 11.14 \mathrm{~Hz}$ ); 5.56 (br dq, $1 \mathrm{H}, \mathrm{H}-6_{\mathrm{ax}}, \mathrm{J} \approx 11.14 \mathrm{~Hz}, J$ $\approx 2.14 \mathrm{~Hz}, J \approx 3.66 \mathrm{~Hz}) ; 3.54\left(\mathrm{~s}, 3 \mathrm{H}, \mathrm{H}-\mathrm{OCH}_{3}\right) ; 3.66(\mathrm{~s}, 3 \mathrm{H}$, $\mathrm{H}-\mathrm{OCH}_{3}$ ); 6.98-7.70 (br m, $20 \mathrm{H}, 2 \times \mathrm{H}-\mathrm{Ar}^{\prime}+3 \times \mathrm{H}-\mathrm{Ar}$ ). ${ }^{13} \mathrm{CNMR}$ (100 MHz, acetone- $\left.d_{6}\right)$ : 132.49 (C-2), 48.72 (C-3), 54.25 (C-4), 50.54 (C-5), 55.50 (C-6), 200.70 (C-CO), 202.43 (C-CO), 127.80-159.42 (C-5 × Ar); MS (ApcI): m/z 689/691/693/695 $\left(\mathrm{M}^{+}+1\right)$.

General procedure for the synthesis of 2,6-bis(4-chlorophenyl)-4-(4-methoxyphenyl)pyridine (7a): A mixture of 1,5-diketone $2 \mathbf{a}(1.5 \mathrm{~g}, 0.0035 \mathrm{~mol})$, thioglycollic acid (0.966 g,
$0.0105 \mathrm{~mol})$ and ammonium carbonate $(3.36 \mathrm{~g}, 0.350 \mathrm{~mol})$ in dry benzene $(30 \mathrm{~mL})$ was refluxed for $28 \mathrm{~h}$, collecting the generated water in an azeotropic collector. The reaction mixture was concentrated, extracted with diethyl ether, washed with water until filtrate was neutral and dried over sodium sulphate overnight. Evaporation of the solvent afforded a white solid mass which was recrystallized from acetone-benzene to furnish compound 7a as white crystals, $1.026 \mathrm{~g}$ (87 \%), m.p. 207-208 ${ }^{\circ} \mathrm{C}, \mathrm{R}_{\mathrm{f}} 0.63$ (petroleum ether-diethyl ether, 7:3 v/v); IR (KBr, $\left.\mathrm{cm}^{-1}\right): 1610 v(\mathrm{C}=\mathrm{N}$ and phenyl), 1590, $1570 \mathrm{v}$ (phenyl), 1540, 1505, 1475, 1420, 1375, 1285, 1245, 1165, 1075, 1000, 820. ${ }^{1} \mathrm{H}$ NMR (400 MHz, acetone- $d_{6}$ ): 3.88 (s, 3H, H-OCH ); 8.15 (s, 2H, H-3,5); 7.94 (d, 2H, H-Ar'-2,6, J= 8.85 Hz); 7.10 (d, 2H, H-Ar'-3,5, $J=8.85 \mathrm{~Hz}$ ); 8.35 (d, 4H, H-2, Ar-2,6, $J=8.69$ ); 7.55 (d, 4H, H-2, Ar-3,5, J=8.69). ${ }^{13} \mathrm{C}$ NMR (100 MHz, acetone- 
$\left.d_{6}\right): 55.82\left(\mathrm{C}-\mathrm{OCH}_{3}\right), 156.86(\mathrm{C}-2,6), 117.24(\mathrm{C}-3,5) ; 129.48$ (C-Ar'-2,6); 115.45 (C-Ar'-3,5), 151.05 (C-4), $131.31\left(\mathrm{C}-\mathrm{Ar}^{\prime}-\right.$ 1), 161.96 (C-Ar'-4), 129.65 (C-2 Ar-2,6), 129.59 (C-2 Ar-3,5), 139.13 (C-2 Ar-1), 135.60 (C-2 Ar-4); MS (DCI): 406/408/ $410\left(\mathrm{M}^{+}+1\right)$; Anal. Calcd for $\mathrm{C}_{24} \mathrm{H}_{17} \mathrm{NOCl}_{2}: \mathrm{C}, 70.95 ; \mathrm{H}, 4.22$; N, $3.45 \%$. Found: C, 70.88; H, 4.21, N, $3.47 \%$.

2,4,6-Tris(4-chlorophenyl)pyridine (7b): Yield $89 \%$, m.p. $220-222^{\circ} \mathrm{C}, \mathrm{R}_{\mathrm{f}} 0.78$ (petroleum ether-diethyl ether, 7:3 v/v); $\operatorname{IR}\left(\mathrm{KBr}, \mathrm{cm}^{-1}\right): 1620 \mathrm{v}(\mathrm{C}=\mathrm{N}$ and phenyl $), 1600,1580 \mathrm{v}$ (phenyl), 1540, 1510, 1480, 1420, 1365, 1250, 1225, 1090, 985, 860, 810. ${ }^{1} \mathrm{H}$ NMR (400 MHz, acetone- $d_{6}$ ): 8.22 (s, 2H, H-3,5); 8.06 (d, $2 \mathrm{H}, \mathrm{H}-\mathrm{Ar}^{\prime}-2,6, J=8.70 \mathrm{~Hz}$ ); 7.10 (d, 2H, H-Ar'-3,5, $J=8.70$ $\mathrm{Hz}) ; 8.42$ (d, 4H, H-2, Ar-2,6, J = 8.65); 7.60 (d, 4H, H-2, Ar$3,5, J=8.65) ;{ }^{13} \mathrm{C}$ NMR $\left(100 \mathrm{MHz}\right.$, acetone- $\left.d_{6}\right): 156.92(\mathrm{C}-$ 2,6), 118.70 (C-3,5); 130.52 (C-Ar'-2,6); 116.55 (C-Ar'-3,5), 151.02 (C-4), 129.49 (C-Ar'-1), 147.35 (C-Ar'-4), 130.12 (C2Ar-2,6), 129.89 (C-2Ar-3,5), 140.08 (C-2Ar-1), 136.22 (C2Ar-4); MS (DCI): $m / z$ 410/412/414 $\left(\mathrm{M}^{+}+1\right)$; Anal. calcd for $\mathrm{C}_{23} \mathrm{H}_{14} \mathrm{NCl}_{3}: \mathrm{C}, 67.26 ; \mathrm{H}, 3.44 ; \mathrm{N}, 3.41 \%$. Found: C, 67.37; H, $3.42 ; \mathrm{N}, 3.40 \%$.

in vitro Anticancer activities: The compounds $\mathbf{3 a}, \mathbf{3 b}$ and 7a have been evaluated in the 3-cell line of three types of human cancers: breast (MCF7), Lung (NCI-H460) and CNS (SF-268) in the one dose primary anticancer assay at $10 \mu \mathrm{M}$ concentration. In the current protocol, each cell line is inoculated and preincubated on a microtiter plate. Test agents were then added at a single concentration and the culture incubated for $48 \mathrm{~h}$. End point determinations were made with alamar blue [20]. Results for each test agent are reported as the percent of growth of the treated cells when compared to the untreated control cells.

in vitro Antimicrobial activities: A total of 4 compounds $(\mathbf{3 a}, \mathbf{3 b}, \mathbf{4} \mathbf{a}$ and $\mathbf{4 b})$ were tested against two Gram +ve bacteria (Staphylococcus aureus, Bacillus substilis) and two Gram -ve bacteria (Estcherichia coli, Pseudomonas aeruginosa) and a yeast, Candida albicans. Sabour and dextrose (SD) and Nutrient broth agar media were obtained from Hi-Media Pvt. Ltd. (Bombay, India) were used for Canadida albicans and test bacteria, respectively. Freshly grown microbial cultures at $37{ }^{\circ} \mathrm{C}$ were appropriately diluted in sterile normal saline solution to obtain a cell suspension of $10^{6} \mathrm{CFU} / \mathrm{mL}$.

Each synthetic compound was dissolved in DMSO to obtain a stock solution of different concentration ranging from $2500 \mu \mathrm{g} / \mathrm{mL}$. The agar well diffusion method [21,22] was used. $0.1 \mathrm{~mL}$ of the diluted inoculum $\left(10^{6} \mathrm{CFU} / \mathrm{mL}\right)$ of test organism was spread on nutrient agar/sabour and dextrose agar plates. Wells of $8 \mathrm{~mm}$ diameter were punctured into the agar medium and filled with $100 \mu \mathrm{L}$ of compound, solvent blank and an antibiotic (chloramphenicol, $100 \mu \mathrm{g} / \mathrm{mL}$ ) to which the test bacteria were sensitive. Fluconozole at the concentration of $100 \mu \mathrm{g} / \mathrm{mL}$ was used as the control against Candida albicans. The plates were incubated for $18 \mathrm{~h}$ at $37{ }^{\circ} \mathrm{C}$. Antimicrobial activity was evaluated by measuring the zone of inhibition against the test organism.

\section{RESULTS AND DISCUSSION}

The aim of the present work was to synthesize dithiazolidin4-one derivatives from chalcones via the formation of 1,5diketones by Michael addition of acetophenone, using thioglycollic acid in the presence of ammonium carbonate. Interestingly, during the preparation of 1,5-diketones ( $\mathbf{2} \mathbf{a}$ and $\mathbf{2} \mathbf{b}$ ) from chalcones (1a and $\mathbf{1 b}$ ) by the Michael addition of $p$-chloroacetophenone in the presence of $\mathrm{NaOH}$ (molar ratio, 1:1:10), a novel cyclohexanol derivative was obtained as a side product (Scheme-I).

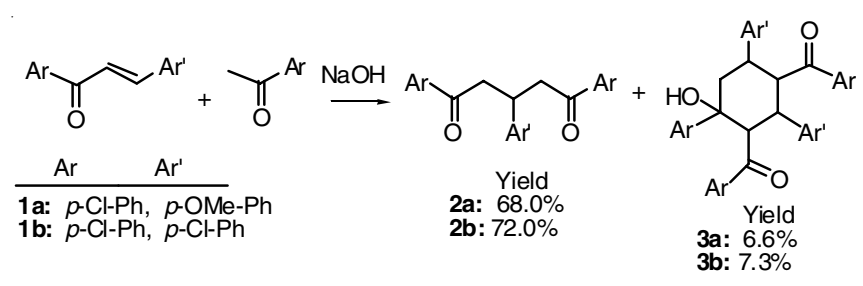

Scheme-I: Synthesis of cyclohexanol derivatives (3a and $\mathbf{3 b})$

It seems that the formation of cyclohexanol in the above reactions has occurred probably by double Michael addition [23] followed by intramolecular aldol condensation [24] of bis-adduct formed in situ. This was confirmed by carrying out Michael addition reaction of the isolated 1,5-diketone 2a to chalcone 1a under the same condition, which yielded cyclohexanol 4a as the sole product. Keeping in view of the novelty of the side product, the same reaction was repeated with different molar ratios of chalcone-acetophenone-sodium hydroxide to set the optimum condition to obtain maximum yield of cyclohexanol and thus the ratio was fixed as 2:1:10 (Table-4).

The dehydration of cyclohexanols $\mathbf{3 a}$ and $\mathbf{3 b}$ with catalytic amount of $p-\mathrm{TsOH}$ resulted in an unusual stereo selective synelimination producing quantitative yields of $\beta, \gamma$-unsaturated cyclohexenes $\mathbf{4 a}(94.4 \%)$ and $\mathbf{4 b}(93.4 \%)$ instead of apparently more stable $\alpha, \beta$-unsaturated cyclohexenes $5 \mathbf{a}$ and $\mathbf{5 b}$ (SchemeII).

This is probably due to the fact that the hydroxyl group in cyclohexanol being in axial disposition, the elimination went

TABLE-4

YIELDS OF COMPOUNDS 2a AND 3a WITH INCREASE IN MOLAR RATIOS OF CHALCONE-ACETOPHENONE

\begin{tabular}{|c|c|c|c|c|c|}
\hline \multirow{2}{*}{ Substrate (chalcone) } & \multicolumn{2}{|c|}{ Chalcone-acetophenone } & \multirow{2}{*}{ Time (h) } & \multicolumn{2}{|c|}{ Products* (\% Yields-Isolated) } \\
\hline & $\mathrm{mmol}$ & Molar ratio & & $2 \mathbf{a}$ & $\mathbf{3 a}$ \\
\hline \multirow{5}{*}{ 1a } & $1.83: 1.83$ & $1: 1$ & 5 & 68 & 6.6 \\
\hline & $2.29: 1.83$ & $1.25: 1$ & 5 & 42 & 30 \\
\hline & $2.75: 1.83$ & $1.5: 1$ & 5 & 23 & 45 \\
\hline & $3.21: 1.83$ & $1.75: 1$ & 5 & 8.4 & 58 \\
\hline & $3.66: 1.83$ & $2: 1$ & 5 & Minor amount & 64 \\
\hline
\end{tabular}

*The yields also vary with the concentration of $\mathrm{NaOH}$. With 5 equivalents, the yield of compound $3 \mathbf{a}$ was low while with 15 equivalents, an undesirable insoluble product was obtained. With 10 equivalents, the maximum yield was obtained. 


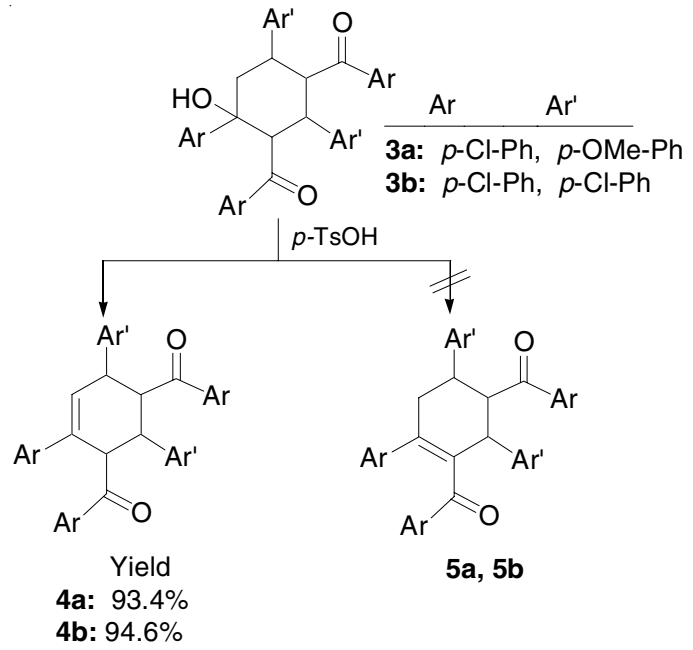

Scheme-II: Synthesis of $\beta, \gamma$-unsaturated cyclohexene derivatives

on that way where there is equatorial hydrogen (cis relationship) required for making up a six-membered transition state for elimination [25]. A plausible mechanism for this may be offered as shown in Scheme-III.

As planned for the synthesis of dithiazolidin-4-ones, the reaction of 1,5-diketone $\mathbf{2 a}$ was carried out with thioglycollic acid in presence of ammonium carbonate (molar ratio 1:5:10)
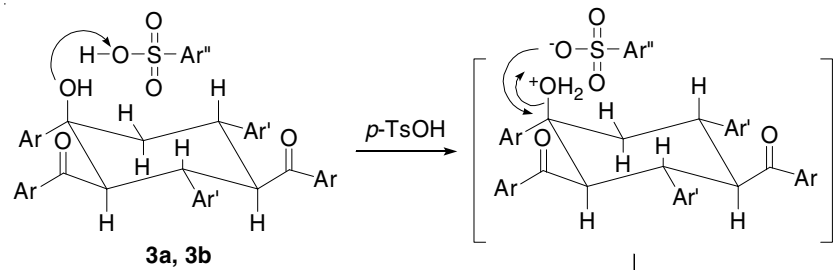

$$
\mathrm{Ar}^{\prime \prime}=\mathrm{p}-\mathrm{Me}-\mathrm{Ph}
$$$$
-\mathrm{H}_{2} \mathrm{O} \downarrow p-\mathrm{TsO}
$$

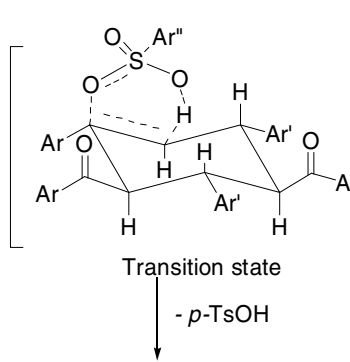<smiles>CC(C)(C)C</smiles>
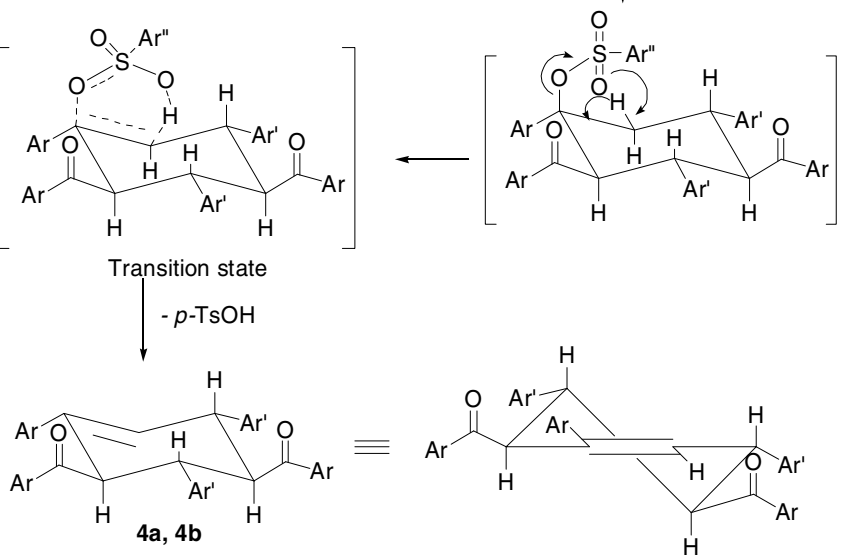

$$
\begin{array}{ll}
\mathrm{Ar} & \mathrm{Ar}^{\prime} \\
\hline \text { a: } & p-\mathrm{Cl}-\mathrm{Ph}, \quad p-\mathrm{OMe}-\mathrm{Ph}
\end{array}
$$

b: $p$-Cl-Ph, $p-\mathrm{Cl}-\mathrm{Ph}$

Scheme-III: A plausible mechanism for the formation of $\beta, \gamma$-unsaturated cyclohexene derivatives in anhydrous benzene, refluxing the reaction mixture for 30 $\mathrm{h}$. The products on crystallization from acetone-benzene yielded an undesired compound, pyridine derivatives $7 \mathbf{a}(87.0 \%)$ and 7b $(89 \%)$ instead of the target compound, dithiazolidin-4ones (6a and 6b) (Scheme-IV).

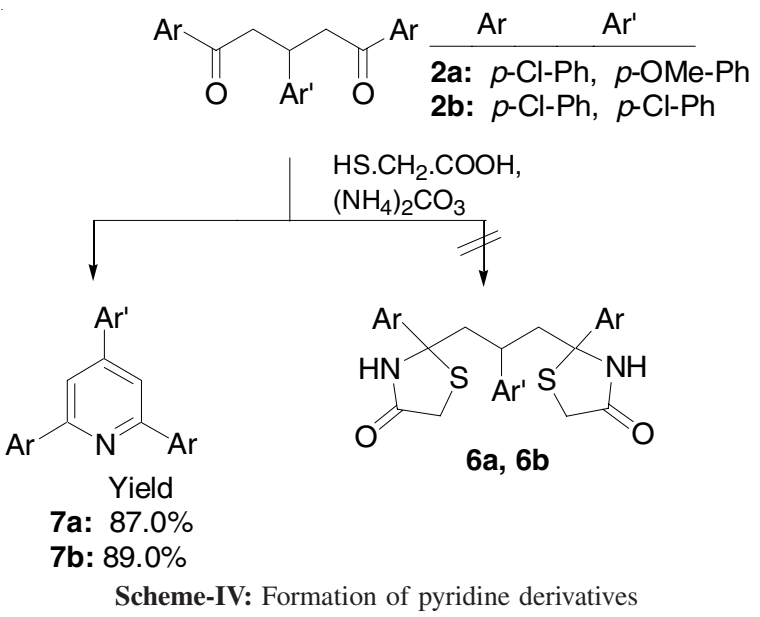

Since, the cyclization of 1,5-diketone with ammonia to form pyridine derivative is a well-known reaction [26,27], it is understood that ammonium carbonate being in excess underwent ring closure with 1,5-diketone prior to the attack with thioglycollic acid. The structures and stereochemistry of the synthesized compounds have been established by the combined study of FTIR, DCI-mass, APcI-mass, ${ }^{1} \mathrm{H}$ NMR, ${ }^{13} \mathrm{C}$ NMR, ${ }^{1} \mathrm{H}-{ }^{1} \mathrm{H}-\mathrm{COSY}$, HETCOR and long-range HETCOR spectra. The assignments of all the signals to individual $\mathrm{H}$ or $\mathrm{C}$ atoms (Tables 1-3) have been performed from their typical chemical shift values, coupling constants and relative integrations.

The coupling constants of compound $\mathbf{3 a}$ (Table-1) indicated that all hydrogen atoms are in axial positions and mutually anti-periplanar except the diastereotopic $\mathrm{CH}_{2}$ hydrogen atoms at $\mathrm{C}-6$ position. From $\mathrm{H}$ and $\mathrm{C}$ values of compound $4 \mathbf{a}$ (Table-3), it was evident that the double bond of cyclohexene ring is not in conjugation with carbonyl group on C-6 $(\alpha, \beta$-unsaturated ketone) as expected, but dehydration went in the other direction affording $\beta, \gamma$-unsaturated ketone.

Anticancer activities: The results of anticancer activity of three compounds $\mathbf{3 a}, \mathbf{3} \mathbf{b}$ and $\mathbf{7} \mathbf{a}$ against 3-cell line of three types of human cancers: breast (MCF7), Lung (NCI-H460) and CNS (SF-268) in the one dose primary anticancer assay are given in Table-5. As results showed in Table-5, all these compounds have not shown any remarkable cytotoxic activity at $10 \mu \mathrm{M}$ concentration.

Antimicrobial activities: The results for antimicrobial activities of the tested compounds detected against the test organisms Escherichia coli, Bacillus subtilis, Staphylococcus

RESULT OF ANTICANCER ACTIVITY OF THE COMPOUNDS AGAINST 3-CELL LINES OF HUMAN CANCERS

\begin{tabular}{ccccccc}
\hline \multirow{2}{*}{ Test compound } & \multirow{2}{*}{ Sample } & \multirow{2}{*}{ Concentration } & \multirow{2}{*}{ Units } & \multicolumn{3}{c}{ Retardation of growth (\%) } \\
\cline { 5 - 7 } & & & & (Breast) MCF7 & (Lung) NCI-H460 & (CNS) SF-268 \\
\hline 3a & 1 & $1.000 \mathrm{E}-04$ & Molar & 72 & 86 & 77 \\
3b & 1 & $1.000 \mathrm{E}-04$ & Molar & 90 & 91 & 53 \\
7a & 1 & $1.000 \mathrm{E}-04$ & Molar & 75 & 81 & 106 \\
\hline
\end{tabular}


TABLE-6

ANTIMICROBIAL ACTIVITY OF COMPOUNDS BY AGAR WELL DIFFUSION METHOD

\begin{tabular}{|c|c|c|c|c|c|c|}
\hline \multirow[b]{2}{*}{ Test compound } & \multirow{2}{*}{$\begin{array}{c}\text { Effective } \\
\text { concentration } \\
(\mu \mathrm{g} / \text { well })\end{array}$} & \multicolumn{5}{|c|}{ Antimicrobial activity in terms of zone of inhibition $(\mathrm{mm})$} \\
\hline & & $\begin{array}{c}\text { Staphylococcus } \\
\text { aureus }\end{array}$ & $\begin{array}{c}\text { Bacillus } \\
\text { subtilis }\end{array}$ & $\begin{array}{l}\text { Escherichia } \\
\text { coli }\end{array}$ & $\begin{array}{c}\text { Pseudomonas } \\
\text { aeruginosa }\end{array}$ & $\begin{array}{l}\text { Candida } \\
\text { albicans }\end{array}$ \\
\hline 3a & 250 & - & - & 19 & 13 & - \\
\hline 3b & 250 & - & - & 20 & 12 & - \\
\hline $4 a$ & 250 & - & - & 12 & 10 & 12 \\
\hline 4b & 250 & - & - & 20 & 15 & - \\
\hline Chloramphenicol & $100 \mu \mathrm{g} / \mathrm{well}$ & 25 & 20 & 24 & 30 & - \\
\hline Fluconozole & $100 \mu \mathrm{g} /$ well & - & - & - & - & 25 \\
\hline
\end{tabular}

aureus, Pseudomonas aeruginosa and Candida albicans are presented in Table-6. Solvent control (DMSO) showed a nonsignificant inhibition to test microorganisms. Antimicrobial activity against Gram -ve bacteria was deduced in all compounds. However, such activity was not detected in all compounds against Gram +ve bacteria.

The compounds exhibited antimicrobial activity at the concentration of $250 \mu \mathrm{g} / 100 \mu \mathrm{L}$. One compound $4 \mathbf{a}$ also demonstrated antifungal (anticandidal) activity. Collectively, it has been found that all compounds $(\mathbf{3 a}, \mathbf{3 b}, \mathbf{4 a}$ and $\mathbf{4 b})$ showed significant antimicrobial activity against Gram -ve bacterial strains. These compounds may be directly explored in the preparation of topical anti-infective agents.

\section{Conclusion}

The aim of this work was to synthesize dithiazolidin-4one derivatives from chalcones via the formation of 1,5-diketones. During the synthesiz of 1,5-diketones ( $2 \mathbf{a}$ and $\mathbf{2 b}$ ) from chalcones (1a and $\mathbf{1 b}$ ) by the Michael addition of $p$-chloroacetophenone in the presence of $\mathrm{NaOH}$ (molar ratio, 1:1:10), a novel cyclohexanol derivative was obtained as a side product. Keeping in view of the novelty of the side product, the same reaction was repeated with different molar ratios of chalcone-acetophenonesodium hydroxide to set the optimum condition to obtain maximum yield of cyclohexanol and thus the ratio was fixed as 2:1:10. The dehydration of cyclohexanols $\mathbf{3 a}$ and $\mathbf{3 b}$ with catalytic amount of $p$-TsOH resulted in an unusual stereoselective syn-elimination producing quantitative yields of $\beta, \gamma-$ unsaturated cyclohexenes $\mathbf{4 a}(94.4 \%)$ and $\mathbf{4 b}(93.4 \%)$ instead of apparently more stable $\alpha, \beta$-unsaturated cyclohexenes. Compounds $\mathbf{3 a}, \mathbf{3 b}$ and $\mathbf{7 a}$ have not shown any significant anticancer activity against three types of human cancers in the one dose primary anticancer assay. The results of antimicrobial studies showed that all compounds demonstrated significant antimicrobial activity against Gram -ve bacterial strains at the concentration of $250 \mu \mathrm{g} / 100 \mu \mathrm{L}$.

\section{ACKNOWLEDGEMENTS}

The authors acknowledge the financial support for this work from the Deanship of Scientific Research (DSR), University of Tabuk, Tabuk, Saudi Arabia under Grant No. (S-0116-1437). The authors are also thankful to National Cancer Institute (NCI), Bethesda, Maryland, USA for in vitro anticancer screening and Prof. Iqbal Ahmad, Department of Agricultural Microbiology, Aligarh Muslim University, Aligarh, India for performing antimicrobial studies of synthesized compound.

\section{REFERENCES}

1. J. Barluenga, L. Alvarez-Rodrigo, F. Rodriguez, F. Fananas, T. Sordo and P. Campomanes, Angew. Chem. Int. Ed., 46, 2607 (2007); https://doi.org/10.1002/anie.200605172.

2. G. Lu and C. Cai, J. Chem. Res., 35, 147 (2011); https://doi.org/10.3184/174751911X12977065405044.

3. I. Mamedov, R. Abbasoglu, M. Bayramov and A. Maharramov, Magn. Reson. Chem., 54, 315 (2016);

https://doi.org/10.1002/mrc.4377.

4. Y. Obata, C. Jie Li, M. Fujikawa, K. Takayama, H. Sato, K. Higashiyama, K. Isowa and T. Nagai, Int. J. Pharm., 212, 223 (2001); https://doi.org/10.1016/S0378-5173(00)00608-6.

5. S. Pandey, S. Singh, B. Patro and A. Ghosh, Indian J. Chem., 43B, 2705 (2004).

6. L. Rong, X. Wei, Y. Lu and Z. Zong, Youji Huaxue, 32, 1999 (2012); https://doi.org/10.6023/cjoc201204004.

7. G. Rogers, S. Parsons, D. Anderson, L. Nilsson, B. Bahr, W. Kornreich, R. Kaufman, R. Jacobs and B. Kirtman, J. Med. Chem., 32, 1217 (1989); https://doi.org/10.1021/jm00126a013.

8. K. Anan, M. Masui, S. Hara, M. Ohara, M. Kume, S. Yamamoto, S. Shinohara, H. Tsuji, S. Shimada, S. Yagi, N. Hasebe and H. Kai, Bioorg. Med. Chem. Lett., 27, 4194 (2017);

https://doi.org/10.1016/j.bmcl.2017.06.076.

9. A. Dhir, S. Malik, S.V. Kessar, K.N. Singh and S.K. Kulkarni, Eur. Neuropsychopharmacol., 21, 705 (2011); https://doi.org/10.1016/j.euroneuro.2010.12.003.

10. D.C. Kosegarten, J.J. Defeo and D.R. DeFanti, J. Pharm. Sci., 56, 1104 (1967); https://doi.org/10.1002/jps.2600560911.

11. V. Mahyavanshi, S.I. Marjadi and R. Yadav, Arab. J. Chem., 10, S804 (2017); https://doi.org/10.1016/j.arabjc.2012.12.009.

12. H. Parveen, F. Hayat, S. Mukhtar, A. Salahuddin, A. Khan, F. Islam and A. Azam, Eur. J. Med. Chem., 46, 4669 (2011); https://doi.org/10.1016/j.ejmech.2011.05.055.

13. H. Parveen, S. Mukhtar and A. Azam, J. Heterocycl. Chem., 53, 473 (2016); https://doi.org/10.1002/jhet.2427.

14. H. Parveen, R.A.S. Alatawi, N.H. El Sayed, S. Hasan, S. Mukhtar and A.U. Khan, Arab. J. Chem., 10, 1098 (2017); https://doi.org/10.1016/j.arabjc.2015.05.002.

15. S. Mukhtar, M.I. Al-Ahmdi and H. Parveen, Asian J. Chem., 28, 2589 (2016); https://doi.org/10.14233/ajchem.2016.19908.

16. K. Salat, A. Moniczewski and T. Librowski, Mini Rev. Med. Chem., 13, 335 (2013); https://doi.org/10.2174/1389557511313030003.

17. T. Torroba, J. Prakt. Chem., 341, 99 (1999); https://doi.org/10.1002/(SICI)1521-3897(199902)341:2<99::AIDPRAC99>3.0.CO;2-Z.

18. V.P.M. Rahman, S. Mukhtar, W.H. Ansari and G. Lemiere, Eur. J. Med. Chem., 40, 173 (2005); https://doi.org/10.1016/j.ejmech.2004.10.003.

19. S. Mukhtar, M. Rahman, W. Ansari, G. Lemière, A. De Groot and R. Dommisse, Molecules, 4, 232 (1999); https://doi.org/10.3390/40700232.

20. G.D. Gray and E. Wickstrom, Biotechniques, 21, 780 (1996). 
21. C. Perez, M. Pauli and P. Bazerque, Acta Biol. Exp., 15, 113 (1990).

22. A.Z. Beg and I. Ahmad, World J. Microbiol. Biotechnol., 16, 841 (2000); https://doi.org/10.1023/A:1008991724288.

23. A. Lubineau and J. Auge, Tetrahedron Lett., 33, 8073 (1992); https://doi.org/10.1016/S0040-4039(00)74720-4

24. R. Antonioletti, F. Bonadies, E.S. Monteagudo and A. Scettri, Tetrahedron Lett., 32, 5373 (1991);

https://doi.org/10.1016/S0040-4039(00)92389-X.
25. J. March, Advanced Organic Chemistry, John Wiley \& Sons, edn 4, p. 1009 (1992)

26. M. Weiss, J. Am. Chem. Soc., 74, 200 (1952); https://doi.org/10.1021/ja01121a051.

27. F. Krohnke, Synthesis, 1 (1976);

https://doi.org/10.1055/s-1976-23941. 